\title{
Caracterización de las propiedades mecánicas de la madera de balsa (Ochroma Pyramidale) Ecuatoriana
}

\author{
Germán Moncayo, Christian Narváez-Muñoz, Nathaly Villacís, Jorge Melo \\ Departamento de Ciencias de la Energía y Mecánica, Universidad de las Fuerzas Armadas ESPE \\ gamoncayo@espe.edu.ec,cpnarvaez1@espe.edu.ec, cnvillacis@espe.edu.ec
}

\begin{abstract}
Resumen-La madera de balsa ecuatoriana tiene una alta importancia a nivel comercial debido a su peso ligero y sus propiedades mecánicas únicas. En el presente trabajo se realizó un estudio experimental con el fin de determinar las propiedades mecánicas de dicho material de acuerdo a la norma ASTM D143 por lo que se llevaron a cabo ensayos que evalúan las propiedades tales como: módulo de elasticidad longitudinal (MOE), módulo de ruptura (MOR), resistencia a la compresión paralela y perpendicular a la fibra, y resistencia a la cizalla paralela a la fibra, sobre muestras secas con un contenido de humedad aproximado de $12 \%$, las muestras utilizadas fueron clasificadas en las tres clases de densidad internacional $\left(80 \leq 120,120 \leq 180\right.$ y180 $\left.\leq 220 \mathrm{~kg} / \mathrm{m}^{3}\right)$. Además de determinar valores promedio para cada propiedad según la clase a la que pertenece, se describen modelos que relacionan las propiedades mecánicas con la densidad. Así se tiene que el MOE, MOR, resistencia a la compresión paralela y perpendicular a la fibra son proporcionales con la densidad de manera lineal, mientras que la resistencia a la cizalla paralela a la fibra se comporta de manera no lineal.
\end{abstract}

Palabras Claves - Ochroma pyramidale, madera de balsa, densidad, propiedades mecánicas

Abstract-Ecuadorian balsa wood has great commercial relevance due to its low weight and unique mechanical properties. In this work, an experimental study was performed with the main goal of determining the mechanical properties of balsa wood according to ASTM D143. Several tests were conducted under dry samples with an approximate moisture content of $12 \%$ to evaluate properties such as modulus of elasticity (MOE), modulus of rupture (MOR), compressive strength parallel and perpendicular to the fibers, and shear strength parallel to the fibers. The analyzed samples were classified under the three types of international densities $(80 \leq$ $120,120 \leq 180$ y $180 \leq 220 \mathrm{~kg} / \mathrm{m}^{3}$ ). Apart from finding mean values for each property according to its class, models that relate such properties with density are described. In this way, MOE, MOR, and compressive strength parallel and perpendicular to the fibers are directly proportional to the density in a linear way, and shear strength behaves in a nonlinear way.

Keywords - Ochroma pyramidale, balsa Wood, density, mechanical properties.

\section{INTRODUCCIÓN}

La balsa (Ochroma pyramidale) es una de las maderas más ligeras del mundo. Su densidad varía dentro del rango de $40-380 \mathrm{~kg} / \mathrm{m}^{3}$ dependiendo de la edad y hábitat del árbol[1].La microestructura celular de la madera de balsa incluye un volumen muy importante de espacio vacío, lo que hace que tenga una rigidez y resistencia axial específica superior en relación a otros materiales; exhibe además, destacadas características de absorción de energía que se derivan de su relativamente baja densidad. De hecho, puede ser el único material con absorción de energía específica similar a la de los panales metálicos hexagonales de la misma densidad [1]. En lo que a materiales compuestos se refiere, estas propiedades hacen que la madera de balsa sea uno de los materiales más atractivos para el uso como núcleos en estructuras tipo sándwich (estructuras formadas por la adhesión de dos caras o revestimientos relativamente delgados a un núcleo central espeso y de baja densidad), particularmente para aplicaciones marinas. Además tiene aplicaciones en otras industrias comerciales tales como: aeronáutica, transporte, energía eólica [2].

Ecuador es uno de los principales productores y exportadores de balsa; sin embargo existen muy pocas referencias en nuestro país sobre la caracterización de las propiedades mecánicas de esta madera. Se podría utilizar los trabajos de Da Silva \& Kyriakides[1], que determinaron de manera experimental las propiedades mecánicas (elásticas e inelásticas) en las direcciones axial, radial y tangencial en función de la densidad de la madera bajo cargas a compresión, o Kotlarewski et. al [3], que determinó las propiedades mecánicas de la madera de balsa proveniente de Papúa Nueva Guinea, de acuerdo a la norma ASTM D143-09 para aplicarlos en la caracterización de la balsa ecuatoriana.

Es por eso que se llevó a cabo el presente proyecto de investigación, con el objetivo de realizar la caracterización de las propiedades mecánicas de la madera de balsa ecuatoriana, por medio de la realización de ensayos mecánicos (según lo que indica la norma ASTM D143) que permitan determinar las propiedades mecánicas de MOE, MOR, resistencia a la compresión paralela y perpendicular a la fibra, y resistencia a la cizalla paralela a la fibra, de madera de balsa procedente de provincia de Los Ríos, ya que esta área productora abarca el 36\% de la producción de balsa de todo el país, siendo así el principal foco de producción[4].

\section{MÉTODO}

\section{A. Materiales}

Se tomó como referencia la norma ASTM D143 para escoger el número de árboles representativos y la Norma Técnica Colombiana NTC 301 para obtener el número de probetas a ensayar. Se seleccionaron cinco árboles de cuatro años de edad de manera aleatoria procedentes de la provincia de Los Ríos, cantón Valencia, parroquia El Vergel. Los árboles fueron seleccionados y marcados de acuerdo a su buen estado fitosanitario (ausencia de fitóftora o "pata roja” en la base y corazón de agua en el xilema), 
buena rectitud del fuste y escasa presencia de nudos.

\section{B. Preparación de las muestras}

Una vez apeado y troceado el árbol se aplicó emulsión de parafina en los extremos expuestos al aire. El secado de la madera cosechada tomó dos semanas por cada árbol cosechado.En la primera semana de dicho proceso fue secada al aire y los siete días restantes secada al horno, al final del proceso de secado el contenido humedad fue de aproximadamente 12\%. Todos los ensayos mecánicos fueron llevados a cabo en probetas pequeñas libres de defectos. Las muestras fueron secadas hasta que se obtuvo un peso seco al aire constante antes de la prueba. Se midió y pesó todas las muestras inmediatamente antes de la prueba para determinar el volumen de cada muestra, su peso seco al aire, y su densidad.Las pruebas se efectuaron de tal manera que no se produjeron grandes cambios en el contenido de humedad, manteniendo una temperatura entre 20 y $23{ }^{\circ} \mathrm{C}$, como recomienda ASTM D143.Después de cada ensayo, las muestras se colocaron en un horno a $103 \pm 2{ }^{\circ} \mathrm{C}$ durante 24 horas para obtener el peso seco al horno.

\section{Ensayos realizados en las probetas}

Se llevaron a cabo ensayos de flexión estática, compresión paralela a la fibra, compresión perpendicular a la fibra ycizalla paralela a la fibra.

1) Ensayo de flexion estática

Se prepararon especímenes de $25 \times 25 \mathrm{~mm}^{2}$ de sección transversal y longitud de $410 \mathrm{~mm}$. Los ensayos se ejecutaron en una máquina de ensayos universales MTS TK/T5002 con celda de carga tipo S Quantrol A $\pm 1 \mathrm{~N}$. Se aplicó una carga continua a lo largo de toda la prueba a una velocidad de 1,3 $\mathrm{mm} / \mathrm{min}$ hasta que ocurra la falla.

Con los datos del ensayo de flexión estática se obtuvo el MOE de cada probeta, mediante (1).

$$
M O E=\frac{P L^{3}}{48 \cdot \delta_{\max } \cdot I}
$$

Donde $P$ es el valor de carga en la zona elástica, Les la longitud entre apoyos, $\delta_{\max }$ es la deflexión en el centro de la viga eIes la inercia de la sección transversal.

También se obtuvo el MOR de cada probeta (2):

$$
M O R=\frac{M y}{I}
$$

Donde $M$ es el momento flector máximo y y es la distancia del eje neutro a la fibra más alejada.

2) Ensayo de compresión paralela a la fibra

Se realizó en especímenes de $50 \times 50 \mathrm{~mm}^{2}$ de sección transversal y longitud de $200 \mathrm{~mm}$, utilizando una máquina de ensayos universales AMSLER con carga continua a una velocidad de $0.6 \mathrm{~mm} / \mathrm{min}$ hasta que la probeta llegue a la rotura.

Se calculó la resistencia a la compresión paralela a la fibra con (3).

$$
\sigma=\frac{P_{\max }}{A_{t}}
$$

donde $P_{\max }$ es la carga máxima y $A_{t}$ es el área de la sección transversal.
3) Ensayo de compresión perpendicular a la fibra

Los ensayos de compresión perpendicular a la fibra se realizaron en muestras de 50x50 $\mathrm{mm}^{2}$ de sección transversal y longitud de $150 \mathrm{~mm}$, utilizando una placa metálica cuadrada de $50 \mathrm{~mm}$ de lado, ubicada a distancias iguales en cada extremo de la probeta, para aplicar la carga. Se utilizó el equipamiento detallado en el ensayo de flexión estática.

Se obtuvo la resistencia a la compresión perpendicular a la fibra mediante (4).

$$
\sigma=\frac{P}{A_{C}}
$$

donde $P$ es la carga en el límite proporcional y $A_{c}$ el área de contacto entre las superficies de madera y acero.

4) Ensayo de cizalla paralela a la fibra

Los ensayos de cizalla paralela a la fibra se realizaron en especímenes de 50x50x63 mm con una muesca de $20 \mathrm{~mm}$ en la cara de $63 \times 50 \mathrm{~mm}$ para provocar una falla de corte en una superficie de 50x50 mm. Se utilizó el equipamiento detallado en el ensayo de compresión paralela a la fibra.

La resistencia a la cizalla se calculó mediante (5).

$$
\tau=\frac{V_{\max }}{A_{\text {corte }}}
$$

donde $V_{\max }$ es la carga cortante máxima y $A_{\text {corte }}$ es el área de corte.

\section{ANÁlisis DE RESUltados}

\section{Resumen de resultados}

Los valores mostrados en la Tabla I muestran los valores promedio obtenidos en este estudio, la desviación estándar entre paréntesis y el número de especímenes ensayados entre corchetes.

\section{E. Ensayo de flexion estática}

En las Fig. 1 y Fig. 2 se denota que el MOE y el MOR se relacionan de manera lineal y directamente proporcional a la densidad. En la Fig.3, se observa que muestras con un alto $M O E$ generan un alto valor de $M O R$, lo cual también fue reportado previamente por otros autores[3].

TABLA I

RESUMEN DE VALORES OBTENIDOS PARA LOS ENSAYOS DEL PRESENTE ESTUDIO

\begin{tabular}{cccc}
\hline $\begin{array}{c}\text { Clase de } \\
\text { densidad }\end{array}$ & Baja & Media & Alta \\
\hline MOE [MPa] & $\begin{array}{c}1154.2(180.64) \\
{[20]}\end{array}$ & $\begin{array}{c}1929.89 \\
(280.68)[23]\end{array}$ & $\begin{array}{c}2507.25 \\
(286.69)[7]\end{array}$ \\
MOR [MPa] & $8.33(1.12)[20]$ & $\begin{array}{c}15.09(3.07) \\
{[23]}\end{array}$ & $21.63(2.87)[7]$ \\
$\begin{array}{c}\text { Comp. } \\
\text { Paralela a la } \\
\text { fibra [MPa] } \\
\text { Comp. }\end{array}$ & $6.92(1.41)[21]$ & $9.88(1.08)[21]$ & $15.52(2.94)[8]$ \\
$\begin{array}{c}\text { Perpend. a la } \\
\text { fibra [MPa] } \\
\text { Corte [MPa] }\end{array}$ & $0.36(0.11)[21]$ & $0.61(0.13)[16]$ & $0.77(0.09)[13]$ \\
\hline \hline
\end{tabular}


MOE vs Densidad

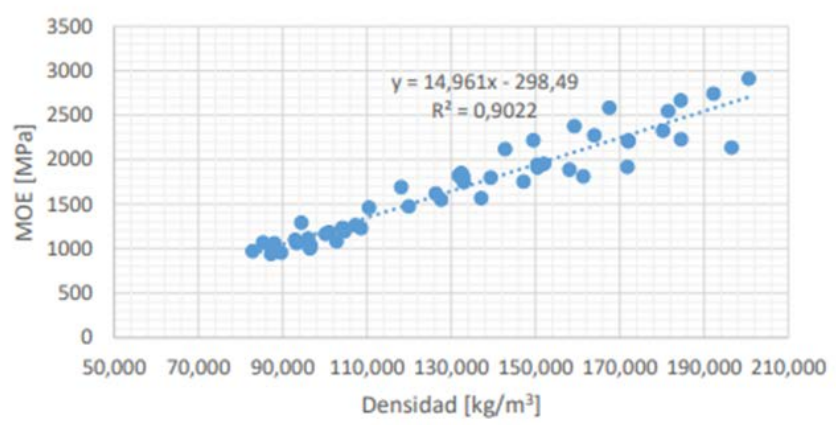

Fig. 1. MOE vs Densidad.

Los valores promedio de MOE y MOR que se presentan en este estudio en la clase baja son similares a los valores mínimos reportados por Wiselius [5]quien utilizó madera procedente de Papúa Nueva Guinea. Los valores máximos obtenidos por el autor antes mencionado son ligeramente inferiores a los registrados en este estudio en la clase media. Por otra parte Kotlarewski et al. [3] evidencia valores similares a los registrados en este trabajo. Los valores de Wiselius y Kotlarewski et al. para MOE fueron de 11551645 MPa y 1222-2037 MPa respectivamente; mientras que para MOR fueron de 8.5-12.5 MPa y 9.83-16.63 MPa. Almeida y Martínez[6], quienes utilizaron madera procedente de Guayas reportan valores para MOE que son mayores a los de este trabajo en 40 y $57 \%$ en las clases media y alta respectivamente.

\section{F. Ensayo de compresión paralela a la fibra}

En la Figura 4 se observa que la resistencia a la compresión paralela a la fibra se relaciona con la densidad a través de una tendencia lineal directamente proporcional.

El valor registrado por Eddowes, mostrado en [3] quien utilizó madera procedente de Islas Salomón se encuentra en el centro del rango de los valores encontrados en este estudio entre la clase baja y alta. Mientras que Kotlarewski et al. [3]presenta valores similares a los de este estudio en las clases media y alta.

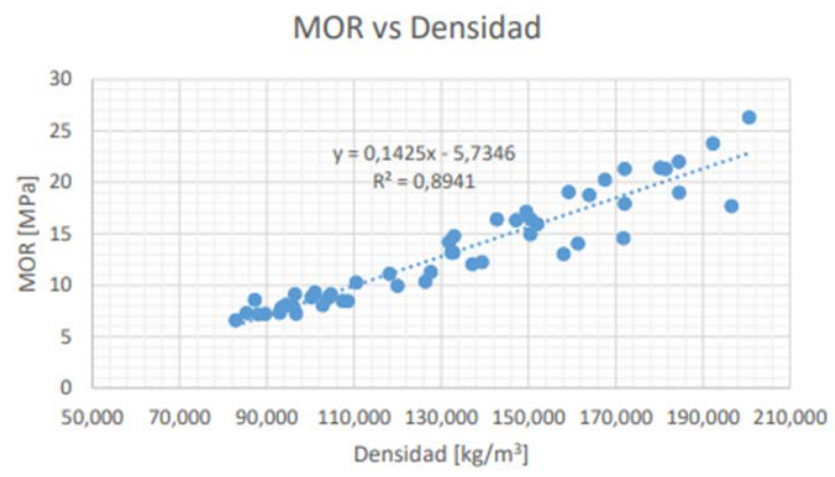

Fig. 2. MOR vs Densidad.

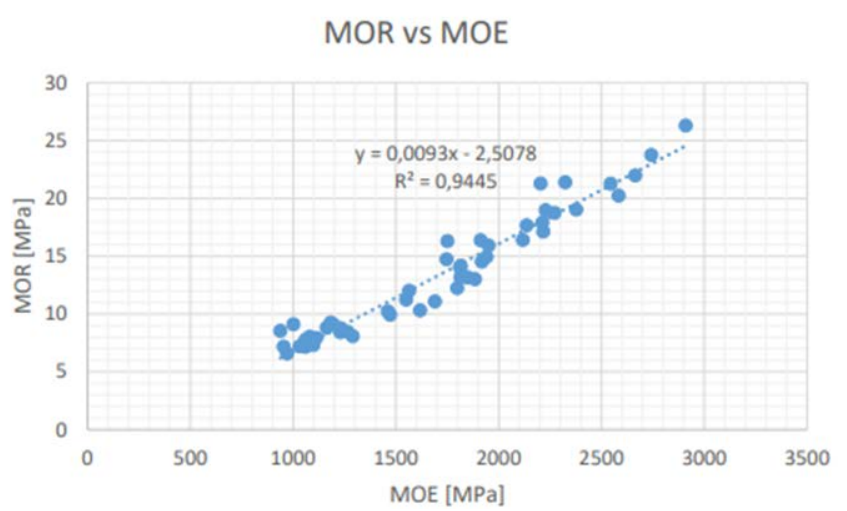

Fig. 3. MOR vs MOE.

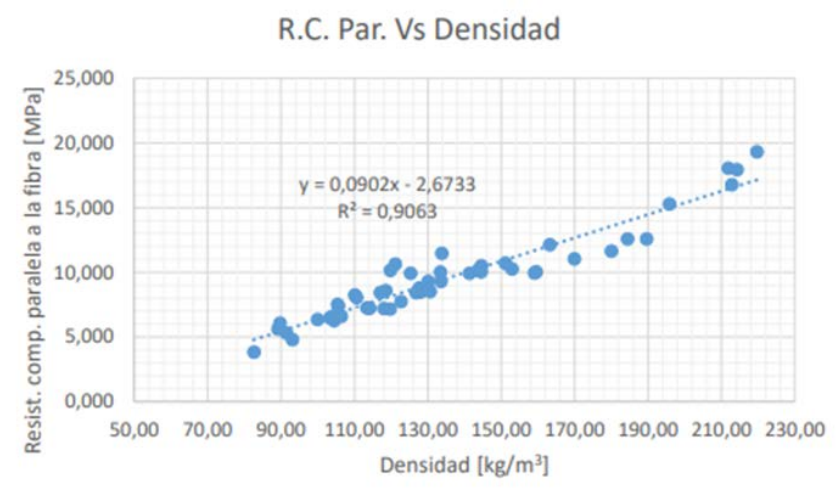

Fig. 4. Resistencia a la compresión paralela a la fibra vs Densidad.

\section{G. Ensayo de compresión perpendicular a la fibra}

Para este ensayo, con fines comparativos al estudio de Kotlarewski et al. [3] se calculó la resistencia a la compresión perpendicular a la fibra a $2.5 \mathrm{~mm}$ de compresión, que para la clase de densidad baja fue de 0.52 $\mathrm{MPa}$, en la clase media fue $0.89 \mathrm{MPa}$, mientras que en la clase de densidad alta fue 1.13 MPa. Kotlarewski et. al[3] presenta valores ligeramente superiores a los de este estudio en 23 y $28 \%$ en las clase baja y media respectivamente.

En la Fig. 5 se observa que la relación resistencia a la compresión perpendicular a la fibra vs densidad tiene una tendencia lineal directamente proporcional.

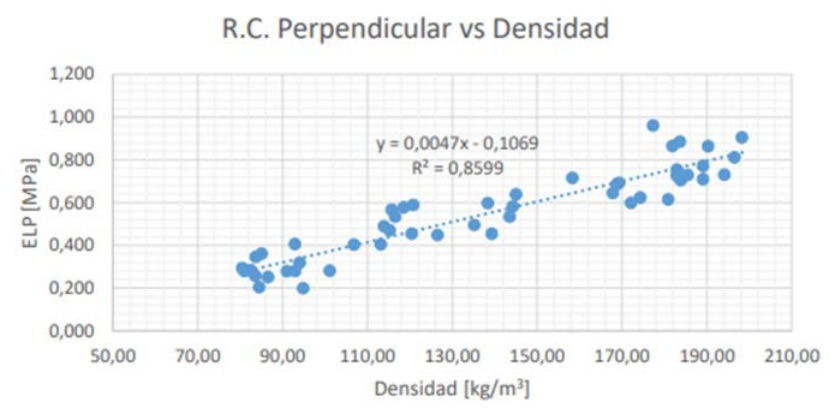

Fig. 5. Resistencia a la compresión perpendicular a la fibra vs Densidad.

\section{H. Ensayo de cizalla paralela a la fibra}

En la Fig. 6 se observa que la relación resistencia a la compresión perpendicular a la fibra vs densidad tiene una tendencia no lineal directamente proporcional. 


\section{R. Cizalla paralela vs Densidad}

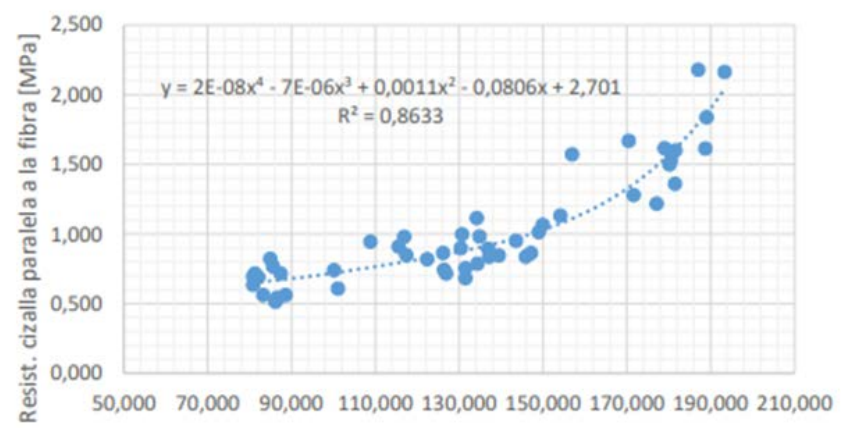

naneidad fLa Im37

Fig. 6. Resistencia a la cizalla paralela a la fibra vs Densidad.

Los valores promedio de resistencia a la cizalla paralela a la fibra que se presentan en este estudio en la clase media y alta son mayores a los valores registrados por Tsoumis [7]; mientras que Kotlarewski et. al[3] presenta valores superiores a los de este estudio en 116 y $90 \%$ en las clases baja y media respectivamente.

\section{CONCLUSIONES}

Para madera de balsa cuya densidad varía en el rango de 80 a $220 \mathrm{~kg} / \mathrm{m}^{3}$, se determinó quelos valores promedio para cada propiedad en las clases de densidad baja, media y alta respectivamente fueron: MOE 1154.2, 1929.9 y 2507.3 MPa, MOR 8.3, 15.1 y $21.6 \mathrm{MPa}$, resistencia a la compresión paralela a la fibra 6.9, 9.9 y $15.5 \mathrm{MPa}$, resistencia a la compresión perpendicular a la fibra 0.36 , 0.61 y $0.77 \mathrm{MPa}$, y resistencia a la cizalla paralela a la fibra 0.7, 1.0 y $1.7 \mathrm{MPa}$. Al hacer un análisis comparativo entre cada una de las clases de densidad y las propiedades mecánicas se determinó que el MOE, el MOR y la resistencia a la compresión paralela y perpendicular a la fibra varían de manera lineal proporcional con respecto a la densidad de la madera de balsa, en tanto que la resistencia a la cizalla tiene un patrón similar excepto porque tiene un comportamiento no lineal. El valor del MOE puede ser útil para predecir el valor de MOR en caso de que no se lleve la probeta hasta la rotura o se utilice algún método de medición alternativo, ya que tienen una relación lineal directamente proporcional. Existe variabilidad entre los resultados conseguidos en este trabajo en comparación a los trabajos de Kotlarewski [3], Almeida y Martínez [6] y ello se debe a las condiciones climáticas (lluvia, viento, entre otras), topografía y suelo del lugar de procedencia de la madera, ya que ello influye en el desarrollo del árbol y por ende sus características físicas y mecánicas.

\section{AGRADECIMIENTOS}

El trabajo no podría haber sido desarrollado sin la colaboración del Laboratorio de Reología y Fluidos Complejos y el Laboratorio de Mecánica de Materiales de la Universidad de las Fuerzas Armadas ESPE.

\section{REFERENCIAS}

[1] A. Da Silva, S. K.-I. J. of S. and Structures, and undefined 2007, "Compressive response and failure of balsa wood," Elsevier.

[2] M. Borrega, L. G.-M. of Materials, and undefined 2015, "Mechanics of balsa (Ochroma pyramidale) wood,” Elsevier.
[3] N. J. Kotlarewski, B. Belleville, B. K. Gusamo, and B. Ozarska, "Mechanical properties of Papua New Guinea balsa wood," Eur. J. Wood Wood Prod., vol. 74, no. 1, pp. 83-89, Jan. 2016.

[4] W. Tene, E. Arias, M. Robles, D. Velasteguí, and S. Cevallos, “Aprovechamiento de Recursos Forestales en el Ecuador,” 2011.

[5] S. Wiselius, "Plant Resources of South-East Asia: timber trees; lesserknown timbers.” Backhuys Publishers, 1998.

[6] F. Almeida and M. Martínez, "Caracterización dinámica de la madera de balsa de Ecuador,” 2017.

[7] G. Tsoumis, Science and technology of wood: structure, properties, utilization. 1991. 